\title{
Fiber Optic Data Bus Using Frequency Division Multiplexing (FDM) and an Asymmetric Coupler
}

\section{H. Zanger and L. Webster}

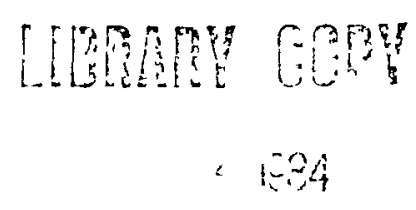

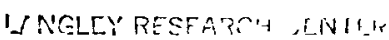

LiDra? ing:

I!..PTCi', VIREITHA 
NASA Technical Memorandum 86015

\section{Fiber Optic Data Bus Using Frequency Division Multiplexing (FDM) and an Asymmetric Coupler}

H Zanger, The City University of New York, Baysıde, New York

L. Webster, Ames Research Center, Moffett Field, Calıfornia

\section{N/SN}

National Aeronautics and

Space Admınıstration 


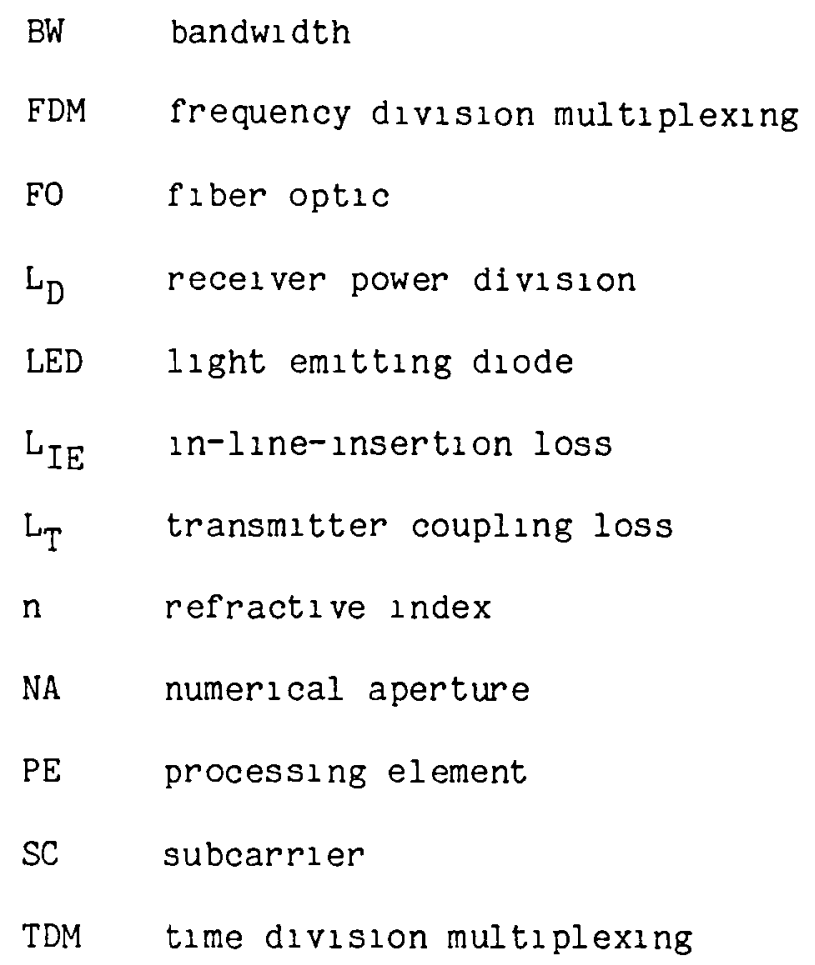


FIBER OPTIC DATA BUS USING FREQUENCY-DIVISION MULTIPLEXING (FDM)

\author{
AND AN ASYMMETRIC COUPLER* \\ H. Zanger** and L. Webster \\ Ames Research Center
}

SUMMARY

\begin{abstract}
A fiber optic data bus, using frequency-division multiplexing (FDM) is discussed. The use of FDM is motivated by the need to avold central control of the bus operation. A major difficulty of such a data bus is introduced by the couplers. An efficient low loss access coupler with an asymmetric structure is presented, and manufacturing processes for the coupler are proposed.
\end{abstract}

\title{
INTRODUCTION
}

A fault-tolerant, distributed, flight-control system has been under development at the NASA Ames Research Center for the past several years (ref. 1). One constraint in the fault tolerant structure required that interelement digital communication was to be accomplished in a manner which maintained the independent and autonomous nature of the redundant elements. A University-NASA cooperative research effort was established to attempt to address the constraint.

Figure 1 shows two types of triple redundant distributed computer systems. The multisuccess paths system ( $f 1 g .1(b)$ ) is to be preferred from a reliability point of view. This system, however, requires a complex communications network. Fiber optics was chosen as the communications medium as it provides for electrical isolation between system elements thereby maintaining their independent property and enhancing system reliability. In addition, time division multiplexing (TDM) for this system has the serious problem of requiring some centralized control. The common element represented by the TDM controller is a single point of failure which cannot be tolerated in a reliable, fault-tolerant system.

A suitable solution to the communications requirement is the use of a fiber optic (FO) data bus employing a frequency division multiplexing (FDM) scheme. The FDM approach complies with the criterion that the system elements be independent as it requires no centralized control. It should be noted that the use of an optical fiber, in addition to its other advantages (ref. 2), offers a large bandwidth (BW). FDM is often considered a BW wasteful approach. This shortcoming, however, is overshadowed by the need for channel independence, and is greatly mitigated by the large $B W$ available in optical fibers. BW efficlency is not necessarlly the most

\footnotetext{
*This work is funded through NASA Grant NCC2-108.

**Queensborough Community College, Electrical Engineering Department, The City University of New York, Bayside, New York 11364.
} 
important consideration in a digital data network, especially if the BW required by the bus users is less than the avallable BW of the communication systems.

The FDM, or any FO data bus, calls for an access coupler with a low insertion loss. The number of bus users is severely $11 \mathrm{mited}$ by the loss introduced into the bus by the access coupler. An efficient coupler is an essential ingredient of any FO data bus.

We greatly appreciate the assistance recelved from Mr. W. Hicks, Polarold Fiber Optics; Dr. W. Sigmond, A-O Scientific Instruments; and Dr. D. Trans, Naval Research Laboratories, in obtaining the specialized fibers and are indebted to them for their advice in the various phases of this project.

\section{THE FIBER OPTIC FDM DATA BUS}

The basic structure of the FO data bus using FDM is shown in figure 2. The FDM subcarriers (SCI, SCJ, SCK) were assigned to allow communications from the processor element in node $k, P E_{k}$, to $P E_{1}$ and from $P E_{j}$ to $P E_{k}$. By increasing the number of detectors in a particular node, the respective $P E$ is made to receive data from any number of other PEs. The structure permits on demand communications from any particular $P E$ to any other PE in the system.

This FDM approach, in essence, replaces the software controls typical in a TDM system, with hardware associated with subcarrier modulation and detection. This hardware-software trade-off is particularly sultable for systems in which communications paths are relatively fixed and can be determined in advance. Most real-time control systems fall into this category.

The use of FDM subcarriers requires the transmission of analog data on the bus, l.e., the subcarriers are analog signals. This is in contrast to the use of digital transmission where the digital data (pulsed signals) directly modulate intensity of the light source. The use of subcarriers implies the transmission of analog data subcarriers rather than the usual digital intensity modulated light as the transmission mode. Consequently, the light source must be relatively linear, otherwise subcarrier harmonics will be generated resulting in channel crosstalk. The analog nature of the data transmission limits the light source to light-emitting diodes (LED) at present since lasers are basically threshold devices and generally not suitable for analog transmission.

\section{THE EXPERIMENTAL BUS}

An FDM data bus (refs. 3 and 4 ) was constructed with two transmit/recelve nodes. The modulation method employed was "frequency shift keyıng"; the subcarriers were $1.08 \mathrm{MHz}$ and $2.35 \mathrm{MHz}(\mathrm{f} 1 \mathrm{~g} .3)$. The system consisted of an asymmetric plastic fiber data bus, with a bus diameter of $500 \mu \mathrm{m}$ and a side arm (access coupler) diameter of $300 \mu \mathrm{m}$ (both outside diameters). Some additional detalis of system performance are given in reference 3. The in-line-insertion loss of the couplers averaged about $3.1 \mathrm{~dB}$. Crosstalk was not noticeable, even though recelver input power levels were as low as $-43 \mathrm{dBm}$. Two factors contributed to the high receiver sensitivity. 
The band pass filter, which is a part of any FDM system, improved the signal-tonolse ratio (band-11mited noise). The use of frequency modulation (FSK) further $1 \mathrm{mproved}$ nolse margins.

It should be noted that an in-line loss of $3.1 \mathrm{~dB}$ is too large to make the data bus useful for more than a few users. An efficient coupler is clearly essential as the following power budget analysis shows.

POWER BUDGET IN BUS

In a data bus structure of the kind investigated (figs, 2 and 3 ), it is important to be able to serve as many users (nodes) as possible. One of the more important parameters affecting the maximum number of nodes that can be served is the in-line-insertion loss, L $L_{I E}$, introduced by each tap. Each tap introduces losses to power passing through the main bus. Since this type of loss is cumulative (more taps means more loss), it ultimately limits the number of taps on the bus. Note that the bus is completely passive; data pass through the bus fiber with no amplification or retransmission. However, the structure shown allows easy introduction of repeaters at appropriate points in the $U$ structure. In addition, with some fine tuning, each transmit/recelve node may serve as a repeater. This active approach was avolded for reasons of reliability.

The results of the power budget analysis are shown in figure 4 in terms of the relation between maximum attenuation, transmit to recelve, versus the number of nodes involved. The in-line-insertion loss was used as a parameter. Note that the transmitter coupling loss $\left(L_{T}\right)$ and the receiver power division $\left(L_{D}\right)$ were selected somewhat arbitrarily: $\mathrm{L}_{\mathrm{T}}=-3 \mathrm{~dB}$ and $\mathrm{L}_{\mathrm{D}}=-13 \mathrm{~dB}$. ( $\mathrm{L}_{\mathrm{T}}$ is half-power 10ss; $\mathrm{L}_{\mathrm{D}}$ is $5 \%$ tapping ratio).

As figure 4 indicates, an improvement in $L$ IE from $-0.5 \mathrm{~dB}$ to $-0.2 \mathrm{~dB}$ allows a substantial increase in the number of nodes the bus can serve. For example, if the allowable transmit/recelve attenuation is $35 \mathrm{~dB}$, a bus with $L_{I E}=-0.2 \mathrm{~dB}$ will be able to serve almost 30 nodes, whereas with $L_{I E}=-0.5 \mathrm{~dB}$, only about 16 nodes may be served.

\section{IMPROVED DATA ACCESS COUPLERS}

To minimize $\mathrm{L}_{\mathrm{IE}}$, an asymmetric bus tap (coupler) structure was investigated (ref. 5). This "Y" structure is shown in figure 5(a). Here, both the diameter and the refractive index $(n)$ of the two fibers differ. The effect of making $n_{T}>n_{B}$ ( $n_{T}$ is the side-arm index, $n_{B}$ is the bus index) is to allow the angle between the tap and the bus $\theta$ to exceed the bus critical angle $\theta$, which depends on the numerical aperture (NA) of the bus fiber. Flgure $5(b)$ is a further improvement on the concept shown in figure 5(a). Here, a prefocusing of the beam takes place at the interface between $n_{T}$ and $n_{T B}$, where $n_{T B}$ is the index of an interface section of fiber, so that $\mathrm{n}_{\mathrm{T}}\left\langle\mathrm{n}_{\mathrm{TB}}>\mathrm{n}_{\mathrm{B}}\right.$.

Typical communication fibers have $\mathrm{NA}=0.22$ and the maximum launch angle is $12.7^{\circ}$. It is not practical to construct a "Y" tap with $\theta \leqq 12.7^{\circ}$. If, however, an interface section is introduced such that, for example, $\mathrm{n}_{\mathrm{TB}}=1.9$, and assuming 
$\mathrm{n}_{\mathrm{B}}=1.4$ and $\mathrm{n}_{\mathrm{T}}=1.4$ (a fiber with a core index of about 1.9 was produced using the SF58 glass made by Schott Optical Glass, Inc.), then the effective launch angle into the bus fiber is increased to $22^{\circ}$, making construction feasible. The angle $\theta$ for full power coupling in the case of $n_{B}=n_{T}$, where the $N A$ of the bus and the tap are the same, can be approximated, for small angles, by

$$
\theta=\left(1-n_{B} / n_{T B}\right) \times 90^{\circ}
$$

\section{MANUFACTURING METHODS}

To facilitate the construction of the coupler, a small indentation is made in the bus fiber ( $f 1 g .6$ ). This places the interface between the two fibers inside the bus fiber. It is then possible to use a bonding agent with an index of refraction matched to the bus fiber cladding rather than to $n_{T B}$, which is much higher. The alternate approach of simply bonding the tap fiber to the bus (after removal of some of the cladding) requires the use of a bonding agent with a refractive index matched to $\mathrm{n}_{\mathrm{TB}}$ (relatively high--about 1.9 or higher). Such cements are not readily avallable and are substantially more opaque, resulting in increased light loss. (Some examples are Cargille Inc. Melt mount code 5870, $\mathrm{n}=1.704$, light yellow; refractive index liquid series $\mathrm{H}, \mathrm{n}=1.9$, yellow to dark brown).

Two methods have been used to produce the indentation. The first involves laser machining and the second uses chemical etching. The laser machining approach uses indirect heating of the glass fiber and resulted in a crevice somewhat larger than desired with a relatively fine finish. The chemical etching has the disadvantage of producing a relatively unpolished surface, leading to increased loss. The two methods can be combined so that the crevice produced by chemlcal etching can be polished using laser techniques.

\section{OTHER APPLICATIONS}

The FDM bus may be used in a number of applications in addition to its use in fault-tolerant, distributed, flight-control systems. Since this approach requires no software controls or an extra hardware controller, it is suitable as a replacement for existing short data lines, such as interbuilding cables or in-plant wirlng. Because of the large fiber BW, the use of the fiber optic cable with FDM will allow easy expansion of data capacities with the addition of simple modulation/demodulation hardware.

The FDM approach is also applicable to communications networks with relatively fixed data paths. Many real-time computer systems fall into this category. Data transmitted from/to a central (or distributed) computer to/from various input/output devices (such as input transducers and controlled devices) can be carried out efficlently using FDM. The use of FDM in video and volce transmission is substantially more efficient than digltized volce (or video) transmission. 


\section{CONCLUSIONS}

The use of FDM in an FO data bus was presented as a solution to the problems of intercomputer communications in a redundant, distributed computer-control system. While FDM may be less efficient in terms of BW utilization when compared to TDM, this consideration is superseded by the need for data channel independence. As part of the bus development, an experimental two-node bus was constructed and relevant data were obtained. One of the difficulties in the bus structure proposed is the in-line-insertion loss of the couplers, as demonstrated by a bus power budget analysis. To overcome this problem, the use of an asymmetric, low-loss coupler is proposed, and manufacturing procedures are suggested.

While the thrust of the research was to solve a particular communication problem in a computer control system, a number of additional applications of the FDM bus are discussed. 
1. Webster; Slykhouse; Booth; Carson; Davis; Howard: Ultrareliable Fault-Tolerant Control Systems--A Conceptual Description. NASA TM-86017, Oct. 1984.

2. Zanger, H.: Interconnections in Real Time, Redundant, Distributed Computing Systems. NASA Ames Cooperative Research Interchange NCA-0R625-001, Aug. 1980.

3. Zanger, H.: Applications of FDM to Fiber Optic Data Bussing in Real Time Redundant, Distributed Computing Systems. NASA Ames Cooperatıve Research Agreement NCC-2-108, Aug. 1981.

4. Zanger, H.: Multiaccess Fiber Optıc Data Bus using FDM/FSK. NASA Ames Cooperatı ve Agreement NCC-2-108, Sept. 1982.

5. Sangyo, M. D.: Japanese Patent no. G02B5/14/HO4B9/00, 1979. 


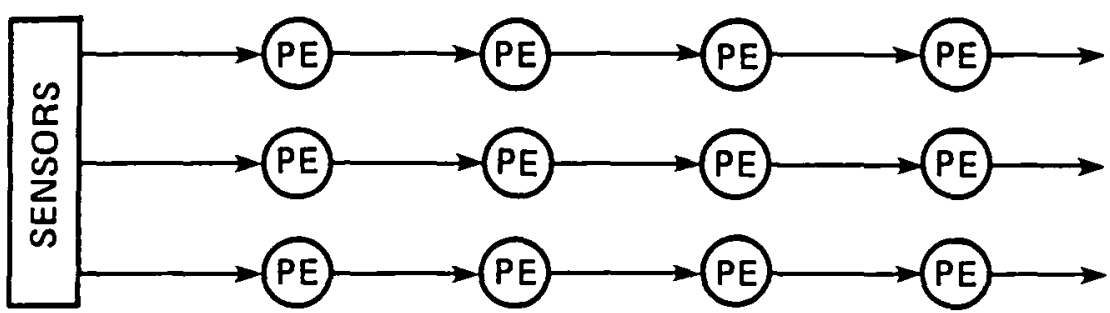

a. SIMPLE REDUNDANCY

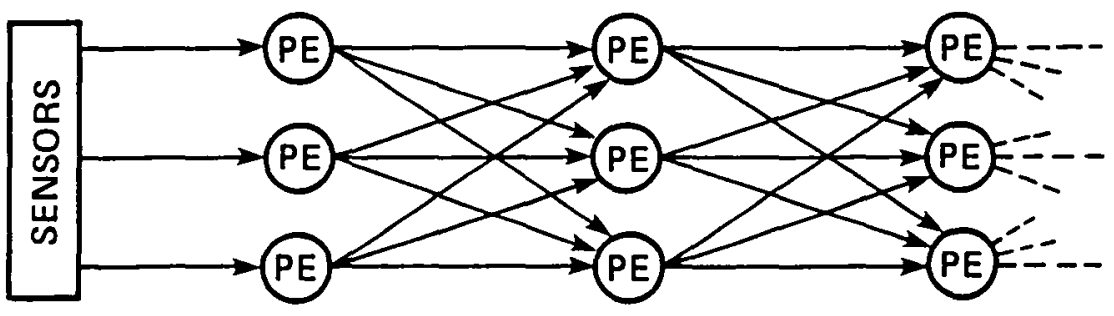

b. MULTIPLE SUCCESS PATHS

Figure 1.- Redundant-distributed systems.

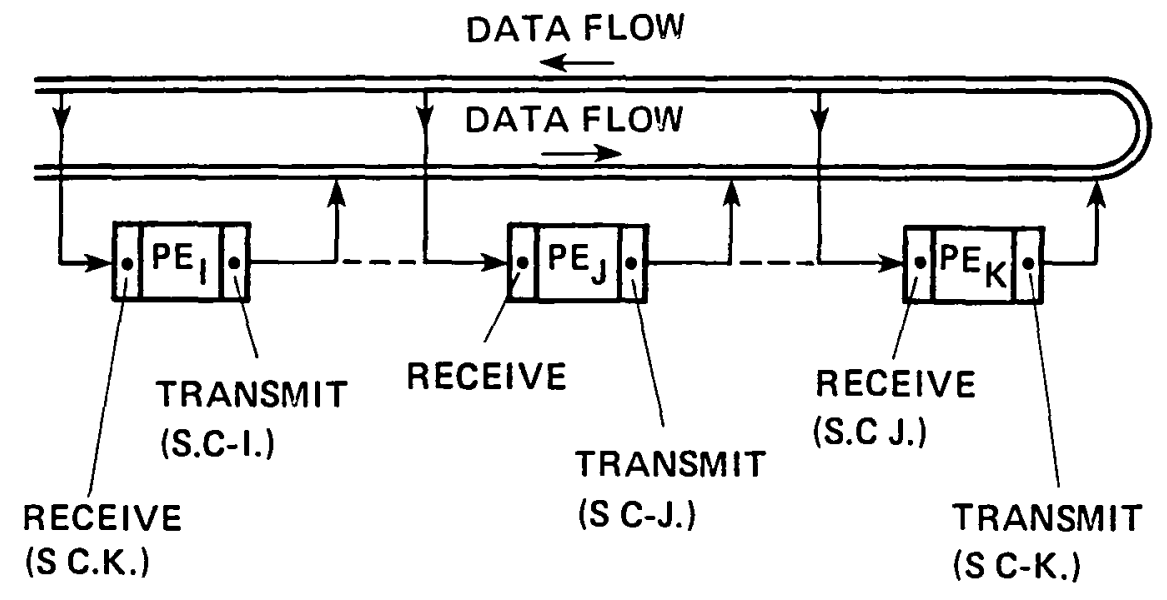

Figure 2.- The FDM bus. 


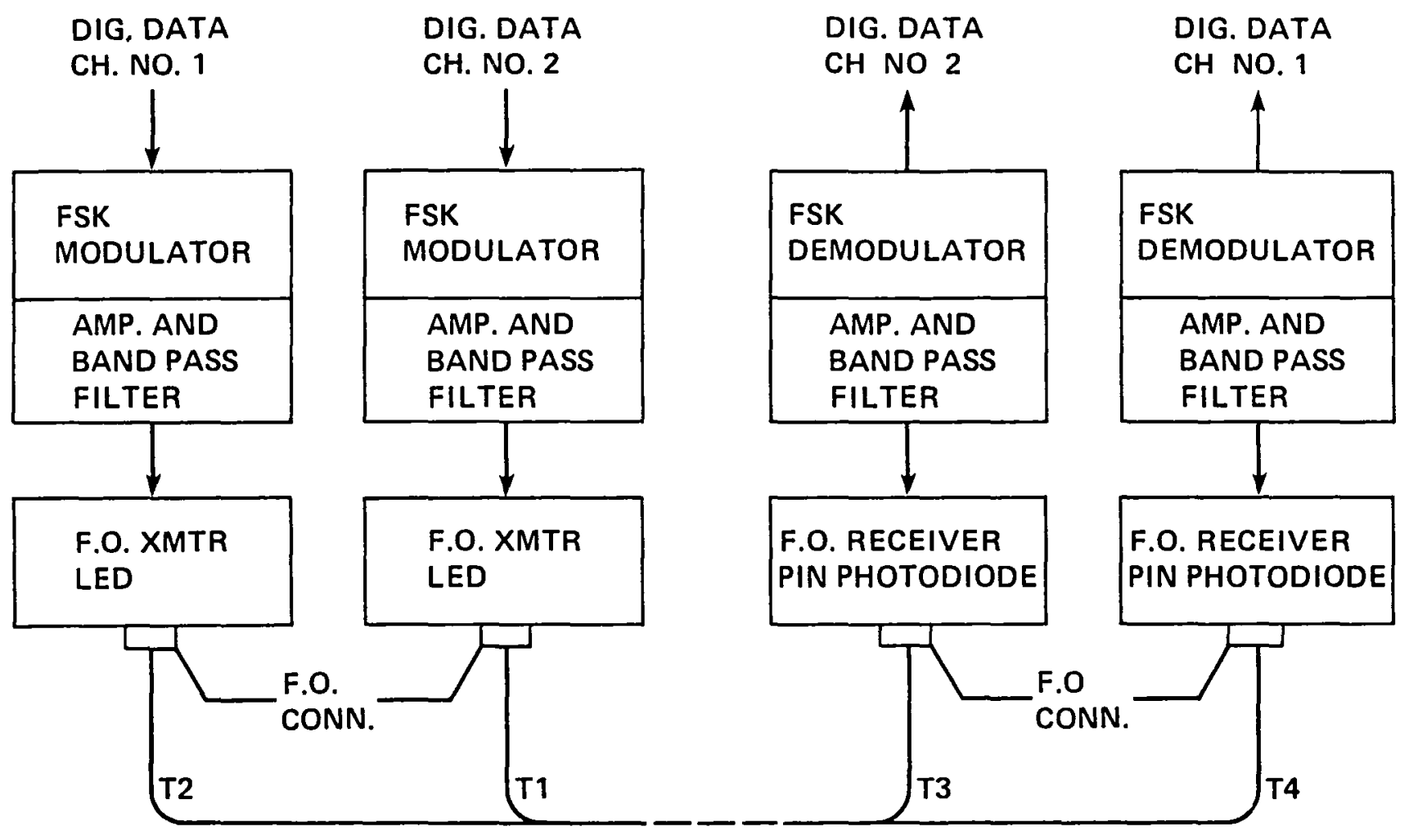

Figure 3.- System diagram. 


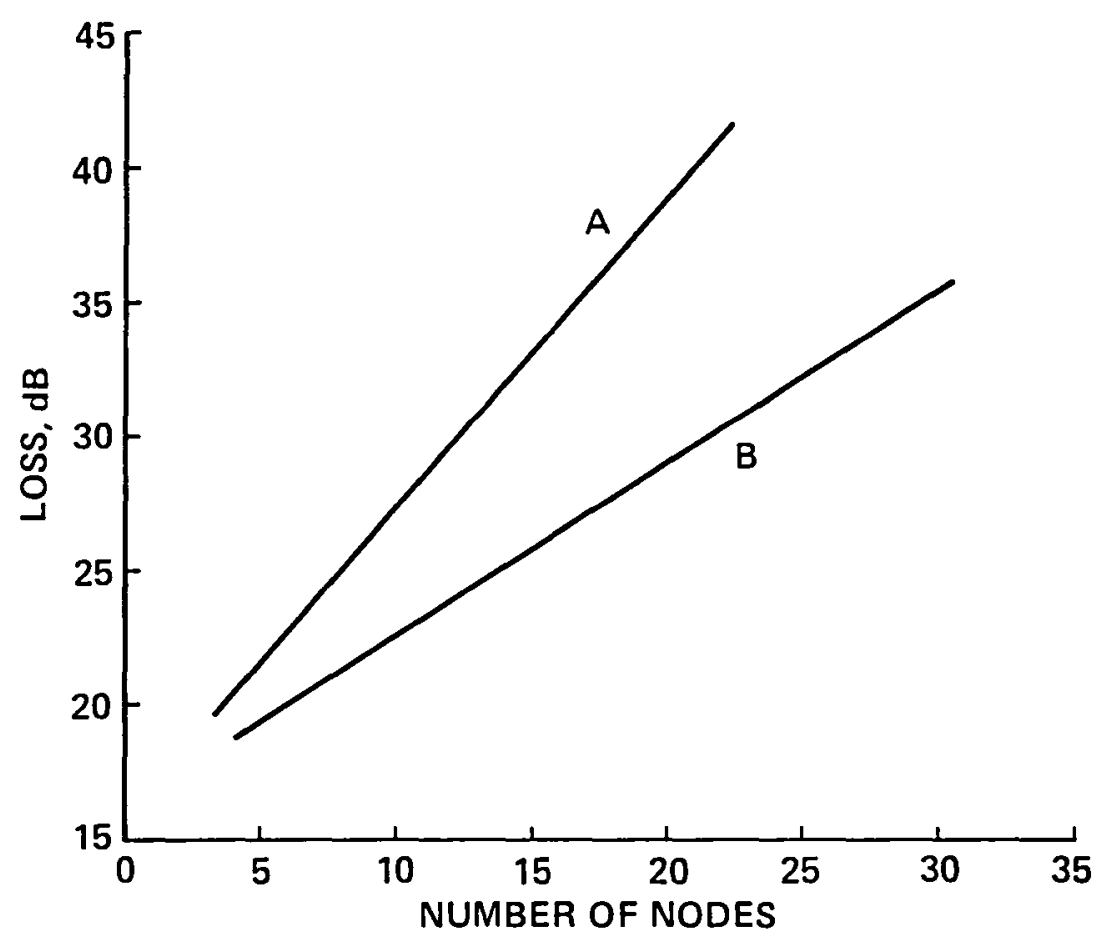

$$
\begin{aligned}
& A-L_{I E}=0.5 \mathrm{~dB} \\
& B-L_{I E}=02 \mathrm{~dB}
\end{aligned}
$$

$$
\begin{aligned}
& L_{I E}=\text { IN-LINE-INSERTION LOSS } \\
& L_{T}=\text { TRANSMITTER COUPLING LOSS } \\
& L_{D}=\text { RECEIVER POWER DIVISION RATIO }
\end{aligned}
$$

Figure 4.- Maximum transmit/recelve loss vs. number of nodes. 
$n_{B}$ BUS

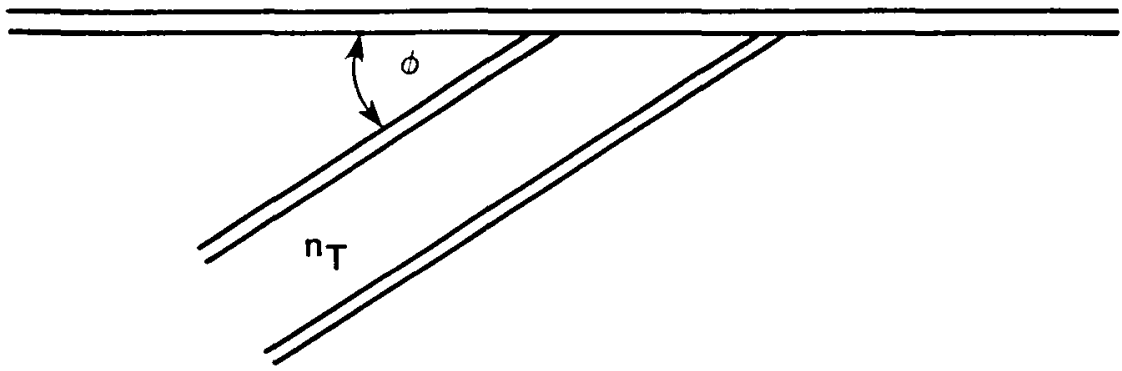

$\mathrm{n}_{\mathrm{B}}<\mathrm{n}_{\mathrm{T}}$

a. WITHOUT INTERFACE SECTION

$n_{B}$ BUS

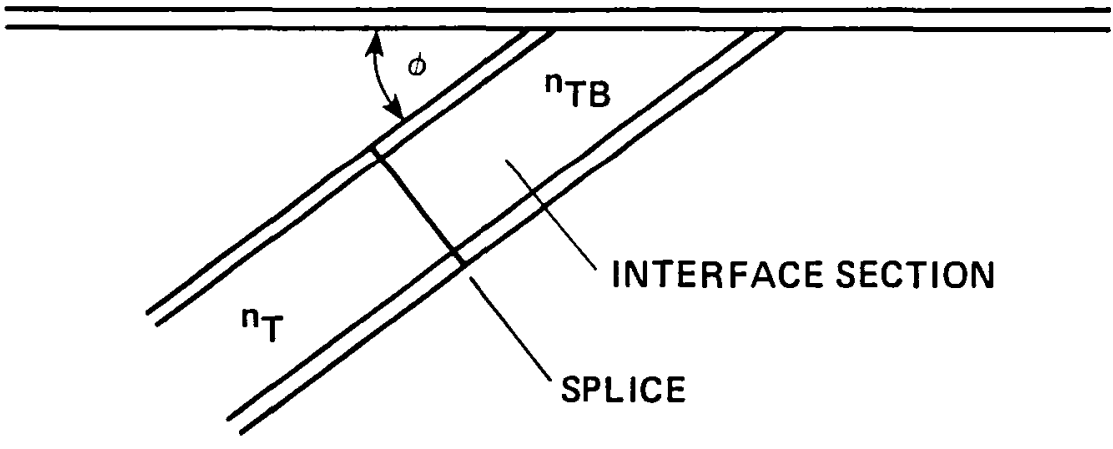

b. WITH INTERFACE SECTION

$$
\mathrm{n}_{\mathrm{B}}<\mathrm{n}_{\mathrm{TB}}>\mathrm{n}_{\mathrm{T}}
$$

Figure 5.- Fo couplers. 


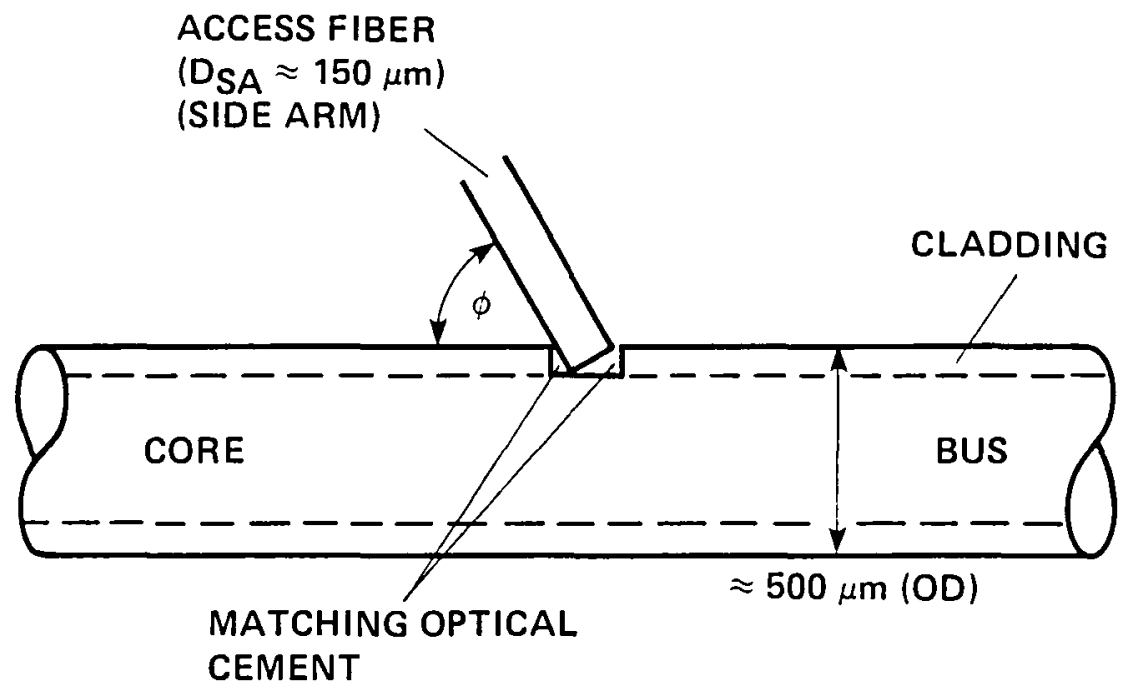

Figure 6.- Proposed manufacturing technique. 


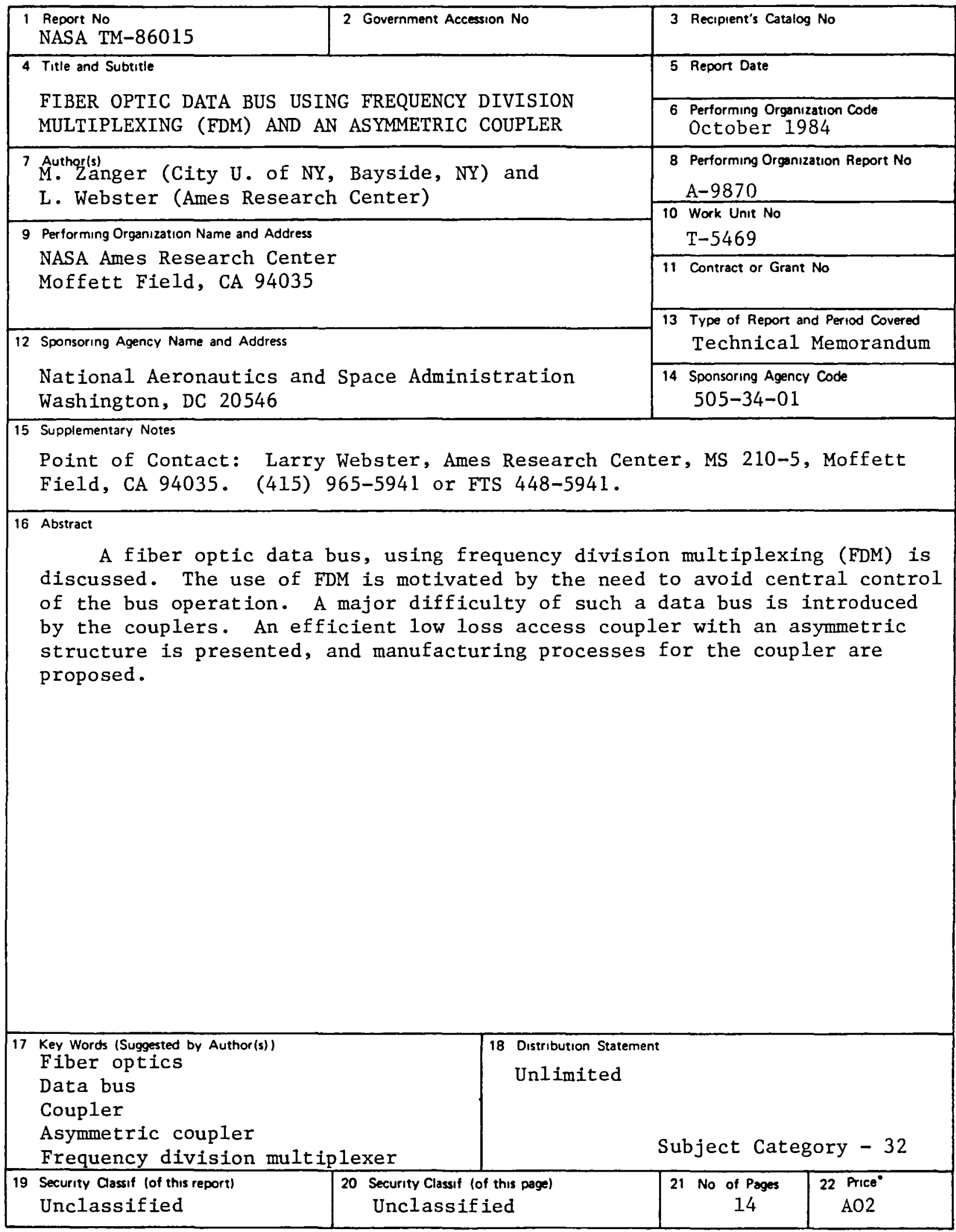


End of Document 